\title{
Heterotrophic Pancreatic Rest Mimicking Tumor in Stomach: A case Report and Literature Review
}

\author{
Waqas Ahmad ${ }^{1 *}$, Jamshed Yousef ${ }^{1}$, Tamer Marei ${ }^{1}$, Maher Nasser ${ }^{1}$ and Mohamed Tahar Yacoubi ${ }^{2}$ \\ ${ }^{1}$ Imam Abdulrahman Al Faisal Hospital, Kingdom of Saudi Arabia \\ ${ }^{2}$ King Abdulaziz Hospital Ministry of National Guard Health Affairs Al Ahsa, Kingdom of Saudi Arabia \\ *Corresponding author: Waqas Ahmad, Imam Abdulrahman Al Faisal Hospital, Kingdom of Saudi Arabia
}

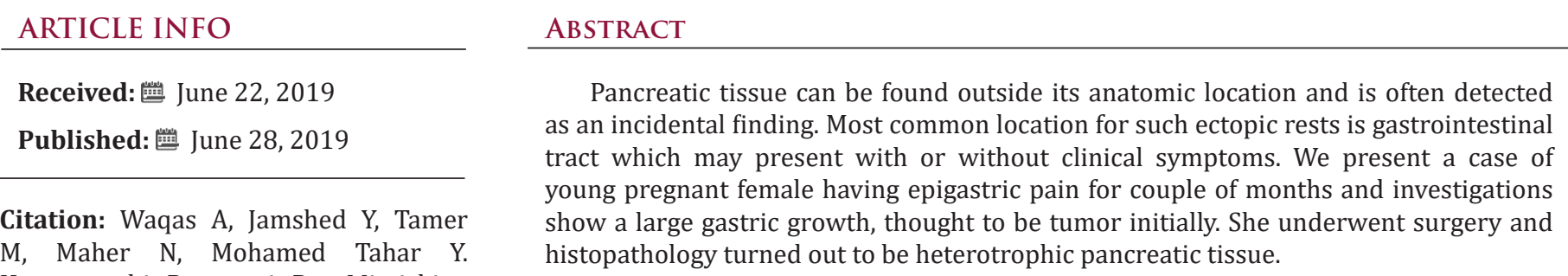

Heterotrophic Pancreatic Rest Mimicking

Tumor in Stomach: A case Report and

Keywords: Pancreatic Tissue; Gastric Mass; Histopathology

Literature Review. Biomed J Sci \& Tech

Res 19(2)-2019. BJSTR. MS.ID.003267.

\section{Introduction}

Pancreatic heterotopia is an entity defined by extra pancreatic tissue without any anatomic or vascular continuity with the pancreas. It can be seen in any part of the gastrointestinal tract with larger percentages found in stomach followed by small intestine. It may remain silent over period of years or present

clinically mimicking symptoms of acid peptic disease, ulcer, upper gastrointestinal tract bleeding, obstruction and even malignant transformation occasionally. Radiological appearances are variable ranging from small lesion to large masses. Histopathological diagnosis remains the gold standard.

\section{Case Report}
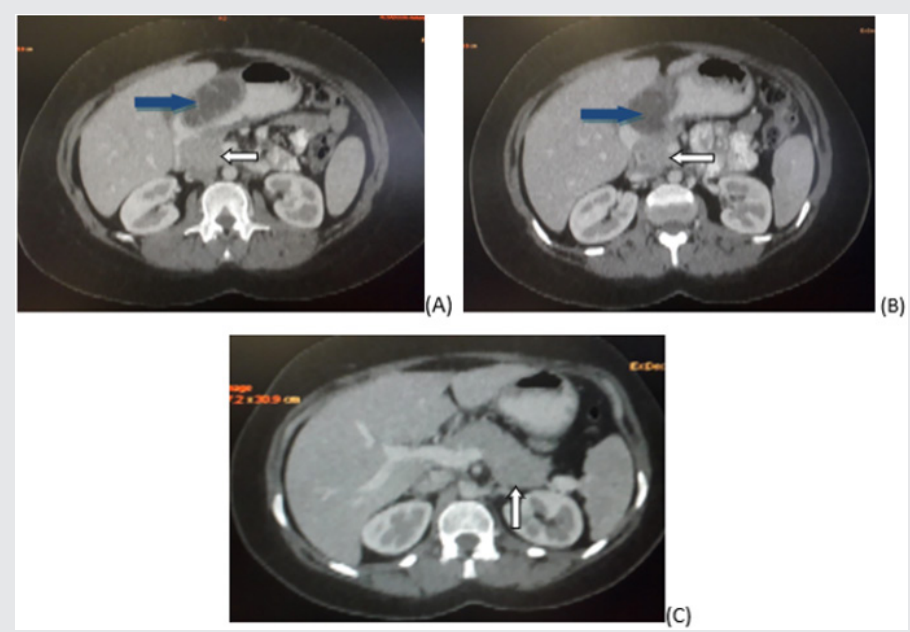

Figure 1: (A, B and C) Axial images of CT abdomen performed with IV and oral contrast shows exophytic cystic density lesion (blue arrows in A \& B) arising from gastric body and pyloric region. Normal pancreas seen in these images (white arrows). 

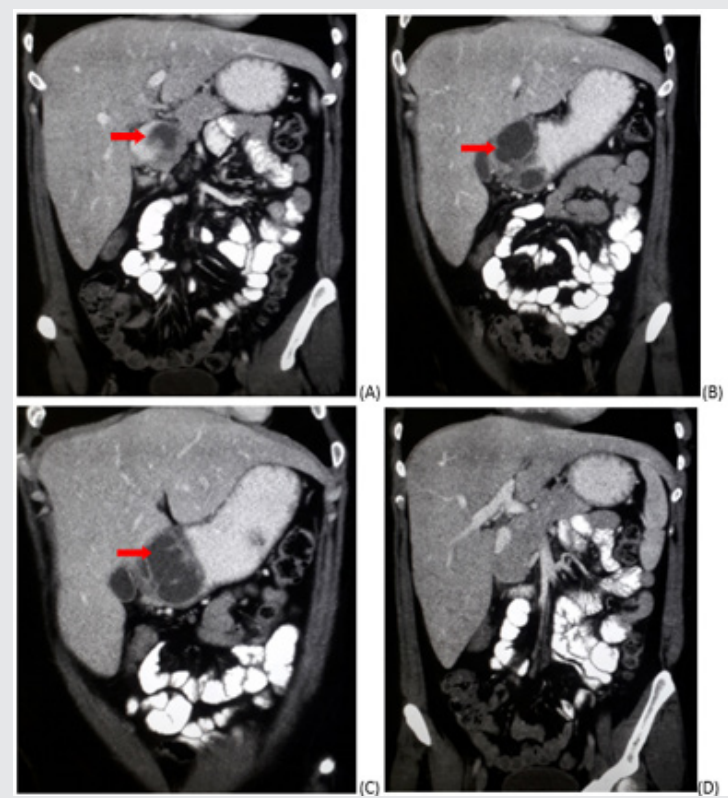

Figure 2: (A, B, C and D) Coronal reformatted CT images with IV and oral contrast showing lesion of cystic density in body and pylorus of stomach (red arrows). Probable diagnosis of GIST was given with such appearances. No obstruction noted. Liver, pancreas and small bowel are unremarkable.
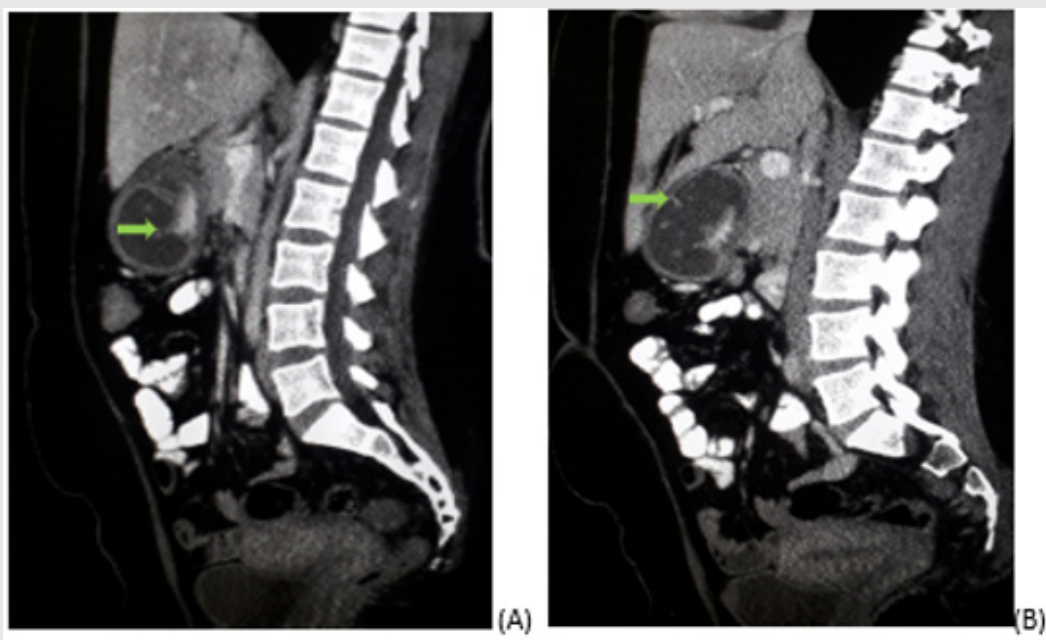

Figure 3: (A and B) Sagittal reformatted images showing same lesion with few internal sepatations (green arrows).

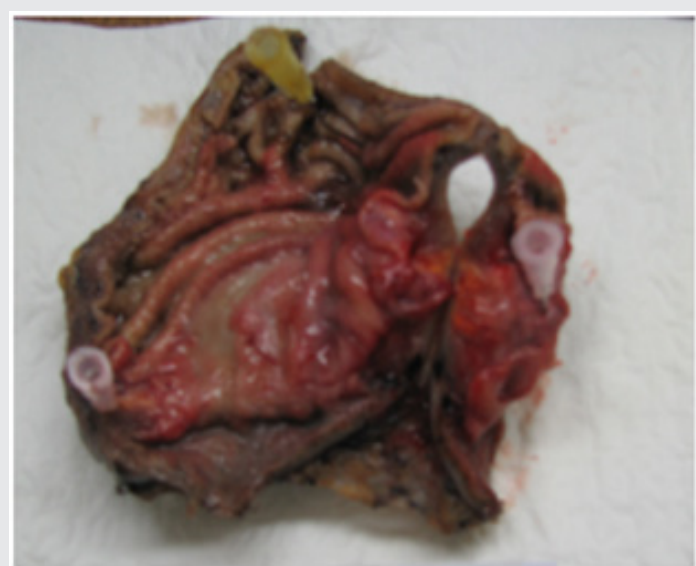

Figure 4: Resected partial gastrectomy surgical specimen received stapled by distal and proximal sides attached to adipose tissue. The mucosa is flattened, presence of area of hyperplastic mucosa. At the cut section, the lesion is ill-defined and showed yellow small nodules involving the wall of the stomach. 

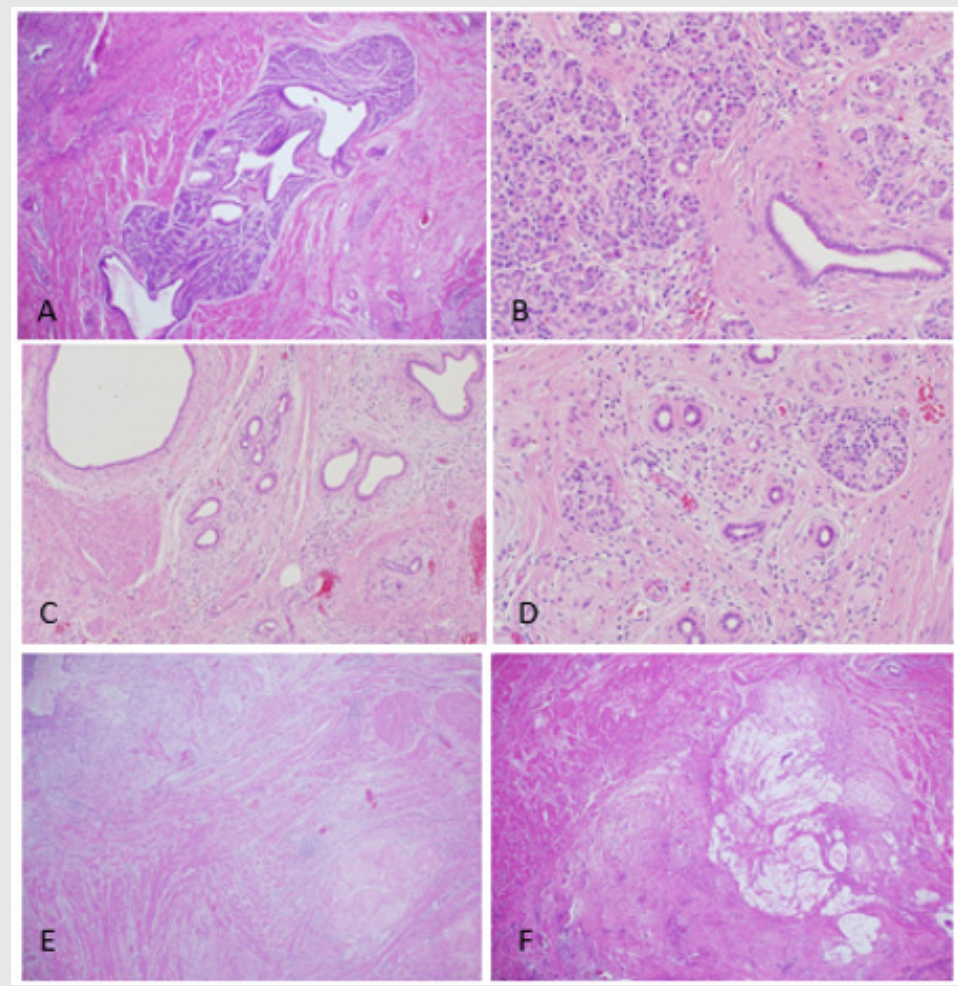

Figure 5: Pancreatic tissue lobules identified in between the muscular propria layers in the gastric wall (A and B). Chronic inflammatory changes with fibrosis of the heterotopic pancreatic tissue (C and D). Areas of tissue necrosis with steatonecrosis close to the pancreatic lobules (E and F).

A 26 year old female presented in outpatient clinic during her second trimester of pregnancy with complaints of daily regular epigastric pain with off and on vomiting. The pain started during pregnancy 2 months back. She never felt such pain before. No melena or hematemesis. Thinking of peptic ulcer she was given proton pump inhibitors which showed no response. Ultrasound abdomen performed was unremarkable. CT abdomen with oral and IV contrast was advised due to her persistent symptoms. Scan showed a large low attenutation soft tissue exophytic lesion with septations and cystic changes arising from the body and pylorus of stomach. No contrast enhancement noted. Considering its size and appearances probable diagnosis of gastrointestinal stromal tumor (GIST) was made (Figures 1-3). Endoscopy done demonstrated sub mucosal origin of the lesion and hence biopsy was not taken. She was referred to surgery department where she had partial distal gastrectomy and gastrojejunal anastomotsis. Histopathology came out to be heterotrophic pancreatic rest rather than neoplastic lesion (Figures 4 \& 5).

\section{Discussion}

Ectopic pancreatic tissue or heterotopic pancreatic tissue, refers to the presence of pancreatic tissue in the submucosal, muscularis or subserosal layers of the gastrointestinal tract outside the normal confines of the pancreas. There is no anatomical or vascular connection with the main pancreas [1]. It can be named as aberrant, accessory pancreas, or sometimes called as pancreatic choristoma and adenomyoma. It is difficult to determine the true incidence as usually patients are asymptomatic and it is detected on autopsy. A prevalence rate of $0.5 \%-13.7 \%$ is quoted in autopsy results, $0.2 \%$ in upper abdominal surgeries as incidental finding and $0.9 \%$ of gastrectomies [2,3]. Gastrointestinal tract is the most common location for this condition specifically stomach followed by duodenum and proximal jejunum. Small percentages include esophagus, ileum, biliary tree, mesentery, and occasionally spleen [4]. Imaging plays role in diagnosis of symptomatic cases, CT being the investigation of choice.

The most common computed tomographic appearance of heterotopic pancreas is usually a small oval intramural mass with microlobulated margins or cystic lesion. The attenuation and enhancement characteristics of these lesions parallel their histologic composition. Acinus-dominant lesions demonstrate avid homogeneous enhancement after intravenous contrast material administration, whereas duct-dominant lesions are hypo vascular and heterogeneous [3]. At times, patients can present with potential complications of heterotopic pancreas which include pancreatitis, pseudocyst formation, malignant degeneration, gastrointestinal bleeding, bowel obstruction, and intussusception [5]. Certain complications may be erroneously diagnosed as malignancy. Endoscopic examination serves the purpose of both local details and tissue specimen. Histopathology remains the gold standard for diagnosis. As far as treatment is considered, small lesions can be removed by endoscopic resection, larger lesions require surgery [6]. 


\section{Conclusion}

Pancreatic heterotopia is a condition which may give deceptive appearances of a gastrointestinal tract tumor especially stomach. Clinician and radiologist should keep it as differential possibility down the line as recognition of imaging features helps in differentiating it from neoplastic conditions and thus in avoiding unnecessary surgery.

\section{References}

1. Brian Gilcrease Garcia, Radswiki (2018) Ectopic pancreatic tissue.

2. Wei R, Wang QB, Chen QH, Liu JS, Zhang B (2011) Upper gastrointestinal tract heterotopic pancreas: findings from $\mathrm{CT}$ and endoscopic imaging with histopathologic correlation. Clin Imaging 35(5): 353-359.

\section{ISSN: 2574-1241}

DOI: $10.26717 /$ BJSTR.2019.19.003267

Waqas Ahmad. Biomed J Sci \& Tech Res

(C) This work is licensed under Creative

Submission Link: https://biomedres.us/submit-manuscript.php
3. Kung JW, Brown A, Kruskal JB, Goldsmith JD, Pedrosa I (2010) Heterotopic pancreas: typical and atypical imaging findings. Clin Radiol 65(5): 403-407.

4. Park SH, Han JK, Choi BI, Kim M, Kim YI, et al. (2000) Heterotopic pancreas of the stomach: CT findings correlated with pathologic findings in six patients. Abdom Imaging 25(2): 119-123.

5. Lai EC, Tompkins RK (1986) Heterotopic pancreas: review of a 26 year experience. Am J Surg 151(6): 697-700.

6. Kojima T, Takahashi H, Parra Blanco A, Kohsen K, Fujita R (1999) Diagnosis of submucosal tumor of the upper GI tract by endoscopic resection. Gastrointest Endosc 50(4): 516-522.

$\begin{array}{ll}\text { BIOMEDICAL } & \text { Assets of Publishing with us } \\ \text { RESEARCHES } & \text { - Global archiving of articles } \\ & \text { - Immediate, unrestricted online access } \\ & \text { - Rigorous Peer Review Process } \\ \end{array}$

